\title{
Oxygen-ozone activity in making factory farms antibiotic-free for prevention of antibiotic resistance
}

\author{
Luigi Valdenassi, ${ }^{1}$ Marianno Franzini, ${ }^{1,2}$ Pierpaolo Garbelli, ${ }^{3}$ Manuele Camolese ${ }^{4}$ \\ ${ }^{1}$ Medical Toxicology, University of Pavia; ${ }^{2}$ Scientific Society of Oxygen Ozone Therapy, Gorle (BG); \\ ${ }^{3}$ Private Veterinarian; ${ }^{4}$ CEO Multiossigen Ozone Technology, Gorle (BG), Italy
}

\begin{abstract}
For some years an increasing number of bacterial strains have shown resistance to common antibiotics and chemotherapy drugs.

The excessive and inappropriate use of antibiotics is at the root of this problem, which could develop to a devastating extent if not controlled properly. It should not be forgotten that the pharmaceutical industry itself appears to be unable to offer the necessary countermeasures in terms of advanced research within tight deadlines because of the objective difficulties with synthesis of new antibiotics.

The use of oxygen-ozone appears to give a concrete answer to the problem through application in the dietary and environment process of livestock.
\end{abstract}

\section{Introduction}

\section{Etiology of resistance}

Recent research has shown that the resistance of Klebsiella to carbapenems in Italy suddenly soared in 2010 before settling at around $30 \%$ of the total of bacterial strains isolated from bacteremia. ${ }^{1}$ However, the more critical resistance is related to Escherichia coli, which expresses resistance to third-generation fluoroquinolones and cephalosporins, Pseudomonas aeruginosa, ceftazidimes and aminoglycosides, and Staphylococcus aureus with a proportion of methicillin-

Correspondence: Marianno Franzini, Scientific Society of Oxygen Ozone Therapy, Via Roma 69, 24020 Gorle (BG), Italy.

E-mail: info@ossigenoozono.it

Key words: Factory farming; 0xygen-ozone; Prevention.

Conflict of interest: the authors declare no potential conflict of interest.

Received for publication: 18 July 2016 .

Accepted for publication: 30 August 2016.

(C) Copyright L. Valdenassi et al., 2016

Licensee PAGEPress, Italy

Ozone Therapy 2016; 1:6274

doi:10.4081/ozone.2016.6274

This article is distributed under the terms of the Creative Commons Attribution Noncommercial License (by-nc 4.0) which permits any noncommercial use, distribution, and reproduction in any medium, provided the original author(s) and source are credited. resistant strains greater than $30 \%$; to these others in progress should be added.

These developments lead us to consider the wide variety of bacterial species present in every environment and the ease with which these can increase in number. When the consistency of a microbial population has values greater than one million individuals, it becomes highly likely that spontaneous events, which modify genetic heritage, will occur in more than one cell. Such mutations could lead the more suitable microorganism to prevail if environmental conditions were to change. But the possibilities of adaptation are virtually limitless considering the number of genes that bacteria have available for finding the most suitable combination to survive under any circumstances. In fact, in microorganisms, unlike in other living species, there can be an exchange of genetic material between different species. ${ }^{2}$ Thus genetic information passes from species to species, making an unlimited assortment of genes available to each individual. This leads to the emergence of strategies for avoiding the lethal action of antibiotics through systems of: i) resistance, when the entire bacterial population ignores the presence of the antibiotic; ii) tolerance, when the entire population survives despite the arrest of growth; iii) persistence, if even a small part of the population survives independently of the action mechanism of the antibiotic used.

\section{Characteristics of chemoresistance}

Because they are able to change their genetic inheritance, microorganisms have virtually the entire set of chromosomal set of all existing species available. This enables bacteria to adapt to any environment, including the one dominated by antibiotics using different mechanisms.

\section{Production of antibiotic-inactivating enzymes}

Lactams are among the most widespread antibiotics in nature and therefore a very present resistance mechanism is the production of lactamase. These enzymes are capable of hydrolyzing the lactam ring of the antibiotic, totally nullifying antibacterial activity. Other enzymes, which, while not hydrolyzing the antibiotic, react with it and change its activities, are aminoglycoside-inactivating enzymes: acetyltransferases, beta-lactamases, phosphotransferases and adenyltransferases. ${ }^{2,3}$

\section{Alteration of permeability of casing}

For many bacteria there is an inherent resistance linked to the fact that hydrophilic or hydrophobic antibiotics may show greater or lesser ease of passing through the casing wall. The penetration of an antibiotic can be reduced by structural changes in the surface of the cell casings. 


\section{Alteration of target}

Substitutions of amino acids by others to make the enzymes more resistant may occur. In this way the target is altered and no longer recognized by the antibiotic itself.

There are many mechanisms that cause target alteration: i) reduced affinity for target; ii) overproduction of target; iii) target replacement; iv) enzymatic modification of target.

\section{Increased efflux}

The antibiotics penetrated in the bacterial cell are turned away from efflux systems, for example in Gram - efflux pumps operate coupled with channel proteins that determine direct ejection of the drug. ${ }^{4}$

It is therefore necessary to reduce the improper use of antibiotics at clinical level, with complete adherence to therapeutic protocols in which humans are involved, but even more in the feeding of livestock, especially when intensive, and in the environment in which these animals live.

It is clear that the antibiotic-resistant bacteria can spread in other animals, in the environment and in humans. The most affected are the subject in the pediatric age range, and they also have the highest incidence of food-borne infections.

\section{Damage arising from the spread of antibiotic resistance}

A modeling study has suggested that an eventual reduction of $30 \%$ in the effectiveness of antibacterial prophylaxis due to antibiotic resistance in people who have to undergo surgery or chemotherapy would cause up to 120,000 infections and 6000 deaths in the United States every year.

Exposure to antibiotics also has prolonged effects on the body; in fact, it can reduce the diversification of intestinal microbiota up to one year after taking antibiotics, also for medium long periods (even for 12 months in the case of ciprofloxacin).

Recent studies have described the association between treatment with beta-lactams and the appearance of strains of $E$. coli resistant to cephalosporins during the life cycle of pigs on farms. ${ }^{4}$

\section{Materials and Methods}

In these observations, 100 seven-day-old piglets were divided into two groups: a control group and one treated with a beta-lactam (ceftiofur).

During fattening, each group was divided into two, administering a treatment with amoxicillin (beta-lactam) in the feed of two of these groups. In this way there were 4 treatment groups: one untreated, one treated with amoxicillin, one with ceftiofur, and one with both antibiotics.

During treatment with ceftiofur, several fecal samples were taken at day 0 , and on days 2, 7, 14, 21 and 42 after treatment; for amoxicillin, on the other hand, sampling was extended up to 73 days after treatment. Isolated samples were analyzed for the presence of genes with resistance to cephalosporins and the plasmids harboring these genes were typified.

\section{Results}

The results suggest that the use of ceftiofur and amoxicillin in various stages of the pig breeding cycle are risk factors for the selection of E. coli cephalosporins resistant. Both beta-lactam antimicrobials selected $E$. coli during the course of the treatments. The same study also showed that the co-selection of resistance genes for different fam- ilies of antimicrobials in the same plasmid is likely to play an important role in the maintenance of distinct mechanisms of resistance. ${ }^{5}$

\section{Discussion}

\section{Hypothesis of oxygen-ozone use for disinfection purposes}

Noted that further studies are necessary for identifying the various risk factors associated with the persistence of resistance mechanisms and minimizing recirculation of resistant strains inside farms, it seemed interesting to see what could occur on farms with a different methodological approach regarding the use of antibiotics.

In this regard, it was considered useful to exploit the disinfection activity exerted by ozone, which, because of its high oxidation potential, is able to intervene on the cellular components of the bacterial cell wall.

It is worth considering that disinfection interventions can be performed in different areas: the air of the environment in which the animals live, drinking water and reconstitution of feed and cleaning water in occupied areas.

Ozone, thus, enters bacterial cells and oxidizes all essential components: enzymes, proteins, DNA and RNA. Practically, it is the most powerful oxidizer available and, precisely through control of its oxidative potential, can be used successfully in industry, agriculture and medicine, obviously monitoring and adapting the level of oxidation used. ${ }^{6}$

Saturating circumscribed environments, it has a powerful antifungal and bactericidal action, and is able to spread on all types of surface.

Some studies have shown that environments with average temperature of $25^{\circ} \mathrm{C}$ and humidity of $80 \%$ and treated with different concentrations of ozone - for example $2 \mathrm{mg}$ of ozone per cubic meter for $120 \mathrm{sec}$ onds - achieve a $70 \%$ reduction of the microbial load in the air and $23 \%$ on surfaces.

With the input of $4 \mathrm{mg}$ for 240 seconds, reduction of the total microbial load rises to $70 \%$ in both the air and on surfaces.

Other studies have found that in a room of $115 \mathrm{~m}^{3}$ treated with ozone for 20 minutes, the bacterial load in the air was reduced by $63 \%$, and that of surfaces by $90 \%$.

The antibacterial action of oxygen-ozone $\left(\mathrm{O}_{2}-\mathrm{O}_{3}\right)$ therapy proves crucial in medicine in the treatment of different diseases characterized by significant stages of infection like trophic ulcers, in which there is the appearance of gram-positive cocci such as $S$. aureus, type B and D streptococci, or anaerobic or gram-bacteria such as E. coli and Klebsiella spp.

In the treatment of intestinal dysbiosis and gastritis from helicobacter pylori, rectal insufflation of hyper-ozonized water has been shown to be effective, thus avoiding the use of antibiotics. ${ }^{7}$

In the antiviral field, ozone has been shown to be capable of modulating, in time dependent doses, the synthesis of immunocompetent cells and an anti-infective defense complementary to the specific defense.

To date, inactivation of viruses has been studied less than that of the bacteria, while being clear that it happens very quickly while requiring a supply of gas at concentrations higher than that for the bacteria. The inactivation curves demonstrate a rapid drop in cultures of up to $99 \%$; the remaining $1 \%$ requires longer for complete inactivation. This behavior is due to the agglutination of viruses with ozone; in fact, methods such as ultrasonic treatment, which eliminate agglutination, would confirm this.

Research conducted on the sensitivity viruses to ozone appear to show that viruses equipped with a membrane are more sensitive than those, which have none. The mechanism of the action of ozone on viruses is not one of destruction but of inactivation. The action of ozone would consist of oxidation with a consequent inactivation of specific viral receptors used for the creation of the bond with the wall of the cell to be invaded. In this way, it is possible to block the mechanism of viral replication at the level of its first phase: cell invasion. 
Based on these observations, it appears useful to follow a new direction regarding the conduct of factory farms, which can fully utilize the antibacterial and antiviral characteristics of $\mathrm{O}_{2}-\mathrm{O}_{3}$, without arousing resistance in animals.

For this purpose, we have started collaboration with a pig farm owned by a veterinarian who is sensitive to these aspects and dissatisfied with massive use of the synthetic chemotherapy drugs normally used in such farms for the control of infectious diseases.

The farm in question is equipped with suitable facilities for using ozone in accordance with the most advanced technologies; in this case, Multiossigen Zoo3 model systems capable of producing ozone levels at very specific concentrations and suitable for the different purposes of these structures. In particular, they have diffusors for the atmosphere to ensure a sustainable hygienic environment for the animals, and dedicated diffusors to convey the required ozone into swill water, which is a fundamental constituent of swine feeding, thus excluding the addition of antibiotics to both feeds and drinking water. The same happens for the water used for cleaning the environments in which pigs are stationed.

This system was put in place in early 2015, then after about a year, even though these are preliminary observations, it can be claimed on the basis of data collected by the Zooprophylactic Institute of Brescia that the structure in question moved to $5 \%$ use of antibiotics compared with previous data and also compared with the average of Brescian farms.

The ozone is insufflated into drinking water and reconstitution of feed, and is supplied in environments according to specific 0xygenOzone Therapy Scientific Society/Multiossigen protocols. On first analysis, this has made it possible to greatly reduce the use of amoxicillin, colistin, lincomycin, tiamulin and tilmicosin. These are all antibiotics, which, with improper use, have caused violent reactions and chemoresistance.

\section{Conclusions}

When it is considered that pigs are essentially weak animals and their immune system is often debilitated, that they frequently encounter infectious diseases of various kinds, but especially of the respiratory tract, it is easy to understand how considerable resistance can spread, with unimaginable consequences given the wide possibility of contagion. In actual application, even if further investigations are required, we can show a performance improvement in terms of animals' quality of life, and relevant economic evidence related to the low running costs of the installations that produce $\mathrm{O}_{2}-\mathrm{O}_{3}$.

\section{References}

1. Van Boeckel TP, Browerb C, Gilbert M. Global trends in antimicrobial use in food animals. PNAS 2015;112:5649-54.

2. Teillant A, Gandra S, Barter S, et al. Potential burden of antibiotic resistance on surgery and cancer chemotherapy antibiotic prophylaxis in the USA: a literature review and modelling study. Lancet Infect Dis 2015;15:1429-37.

3. One Health Initiative. Ministry of Health, Antibiotic resistance, first world week, a one-health approach. 2015. Available from: http://www.onehealthinitiative.com/index.php

4. Cameron-Veas K, Sola'-Gines M, Moreno MA, et al. Impact of the use of -lactam antimicrobials on the emergence of Escherichia coli isolates resistant to cephalosporins under standard pig-rearing conditions. Appl Environ Microbiol 2015;81:1782-7.

5. Burch D. Problems of antibiotic resistance in pigs in the UK. Practice 2005;27:37-43.

6. Kozak GK, Boerlin P, Janecko N. Antimicrobial resistance in Escherichia coli isolates from swine and wild small mammals in the proximity of swine farms and in natural environment in Ontario, Canada. Appl Environ Microbiol 2009;75:559-66.

7. Franzini M, Valdenassi L, Ionita G. First evaluations of oxygenozone therapy in antibiotic-resistant infections. Ozone Ther 2016;1:5-7. 